\title{
The Non-Neighbor Harmonic Index on Elementary Graph Operations
}

\author{
A. Rizwana $^{1^{*}}$, G. Jeyakumar ${ }^{2}$ \\ ${ }^{1}$ Department of Mathematics, Sadakathullah Appa College, Manonmaniam Sundaranar University, Tirunelveli-12, India \\ ${ }^{2}$ Department of Mathematics, St.John's College, Tirunelveli-2, India \\ *Corresponding Author: rijurizwana@gmail.com, Tel.: +91-8508435629
}

Available online at: www.isroset.org

Received:02/Oct/2018, Accepted:25/Oct/2018, Online: 31/Oct/2018

\begin{abstract}
The aim of this paper is to study the behaviour of non-neighbor harmonic index of graphs with respect to the removal of pendant edge and an edge with maximal weight. The non-neighbor harmonic index for the subdivision graphs are computed and discussed in detail.
\end{abstract}

Keywords - non-neighbor harmonic index, elementary graph operations, subdivision graphs.

\section{I-INTRODUCTION}

To bring the power of mathematics to bear on real-world problems, the problem should be first modelled mathematically. Graphs, representatives of mathematics, are remarkable versatile tools for modelling. Graph theory is largely applied to the characterization of chemical structures, as well as to qualitative and quantitative structure-property (QSPR) and structure-activity (QSAR) relations by means of certain numerical characteristics, the topological indices [1]. A single number that can be used to characterize some property of the graph of a molecule is called a topological index. Many topological indices [2,3] have been introduced and studied: Randic index [4], Wiener index [5], First and Second Zagreb indices [6] are a few examples of these concepts. Many mathematical properties of these graph invariants have been studied.

In this paper we are concerned with simple graphs, having no directed or weighted edges, and no self-loops. A graph $\mathrm{G}$ is an ordered pair of two sets $V$ and $E$. The set $V=V(G)$ is a finite non empty set and $E=E(G)$ is a binary relation defined on $V$. A graph can be visualized by representing the elements of $V$ by vertices and joining the pair of vertices $u, v$ by an edge if and only if $u v \in E(G)$. Also we denote $|V(G)|=n$ and $|E(G)|=m$. The degree of the vertex $v \in V(G)$, written $d(v)$, is the number of first neighbors of $v$ in the underlying graph $G$.

Graph operations produce new graphs from initial ones. They may be classified into elementary operations or advanced operations. Elementary operations or editing operations create a new graph from initial one by a simple local change such as addition or deletion of a vertex or of an edge, merging and splitting of vertices or edges. We define $G-u v$ to be the graph obtained from $G$ by deleting the edge $\quad u v \in E(G)$, and $G+u v$ to be the graph that arises from $G$ by adding an edge $u v$ between two nonadjacent vertices $\mathrm{u}$ and $v$ of $G$. The subdivision graph $S(G)$ is the graph obtained from $G$ by replacing each of its edge by a path of length 2 , or equivalently by inserting an additional vertex into each edge of $G$. We use $\mathrm{S}_{\mathrm{n}}, \mathrm{P}_{\mathrm{n}}$ and $\mathrm{K}_{\mathrm{n}}$ to denote the star, path and complete graph on $n$ vertices, respectively. For undefined terminology and notations in the paper, we refer to [7].

The paper is organized as follows, Section I contains the introduction of topological indices, graphs and elementary graph operations. Section II contain the related work of harmonic index and non-neighbor harmonic index, Section III presents the results and discussion of how the non-neighbor harmonic index of a graph $G$ strictly increases and decreases by the removal of pendant edge [8] and an edge with maximal weight respectively. Also the nonneighbor harmonic index for the subdivision graph $S(G)$ is computed for some graphs and the results are discussed in detail. Section IV concludes the research work with the scope for future.

\section{II-RELATED WORK}

In the 1980's, Siemion Fajtlowicz created a vertex-degreebased quantity which was re-introduced by Zhong [9] in 2012 called Harmonic Index [10]. The harmonic index is one of the most important indices in chemical and mathematical fields. The harmonic index gives better correlations with physical and chemical properties of molecules. It is defined as 


$$
H(G)=\sum_{u v \in E(G)}\left[\frac{2}{d(u)+d(v)}\right]
$$

The vertices that are not adjacent to a vertex $v \in V(G)$ are called non-neighbors of the vertex $v$. In this paper we define $\overline{d(v)}$ as the number of the non-neighbors of the vertex $v \in V(G)$, where $\overline{d(v)}=n-1-d(v)$. Based on the non-neighbors of the vertices of a graph $G$ topological indices called Non-Neighbor Zagreb Indices and Non-Neighbor Harmonic index have been introduced [11]. The Non-Neighbor Harmonic Index is defined as

$$
\overline{H(G)}=\sum_{u v \in E(G)}\left(\frac{2}{\overline{d(u)}+\overline{d(v)}}\right)
$$

Non-Neighbor Harmonic Index is considered to be an important Non-Neighbor Topological Index as its values of the Path graph $P_{n}$ is well correlated with the boiling point of the alkanes in Organic Chemistry.

\section{III-RESULTS AND DISCUSSION}

\section{(A) Behaviour of non-neighbor harmonic index by the removal of an edge from the graph.}

In this section, we show that the non-neighbor harmonic index strictly increases by the removal of pendant edge and strictly decreases by the removal of an edge with maximal weight. The graph $K_{n}+e$ is defined and its non-neighbor harmonic index is computed.

Theorem 1.1. Let $G$ be a graph with $n$ vertices and $m$ edges. Removal of pendant edge strictly increases the non-neighbor harmonic index of the graph $G$.

Proof. Let $e=u v$ be a pendant edge of the graph $G$. Let $d(u)=p=1$ and $d(v)=q$ so that $\overline{d(u)}=\bar{p}=n-$ 2 and $\overline{d(v)}=\bar{q} \leq n-3$. Let $x_{1}, x_{2}, \ldots, x_{q-1}$ be the other neighbors of the vertex $v$ with $\overline{d\left(x_{l}\right)}=\bar{q}_{l} \leq n-2$. Let $e_{1}, e_{2}, \ldots, e_{q-1}$ be the edges joining $v$ to $x_{i} \forall i=$ $1,2, \ldots, q-1$. Let $e_{1}^{\prime}, e_{2}^{\prime}, \ldots, e_{\bar{q}}^{\prime}$ be the remaining edges of the graph $G$ with $w_{j}$ and $z_{j}$ as their end vertices. Let $\overline{d\left(w_{J}\right)}=\overline{r_{J}}$ and $\overline{d\left(z_{J}\right)}=\overline{s_{J}}$ for each $1 \leq j \leq \bar{d}$. Here $w_{j}=x_{i}$ and $z_{j}=w_{j}$ for some $j$. Let $G^{\prime}=G-e$ be a connected graph, then

$$
\begin{aligned}
\overline{H(G)}-\overline{H\left(G^{\prime}\right)}= & \frac{2}{n-2+\bar{q}}+\sum_{i=1}^{q-1} \frac{2}{\left(\bar{q}+\overline{q_{l}}\right)}-\sum_{i=1}^{q-1} \frac{2}{\left(\bar{q}+\bar{q}_{l}-1\right)} \\
& +\sum_{j=1}^{\bar{q}} \frac{2}{\left(\overline{r_{j}}+\overline{s_{J}}\right)}-\sum_{j=1} \frac{2}{\left(\overline{r_{j}}+\overline{s_{J}}-2\right)}
\end{aligned}
$$

$$
\begin{aligned}
& =\frac{2}{n-2+\bar{q}}-\sum_{i=1}^{q-1} \frac{2}{\left(\bar{q}+\bar{q}_{l}\right)\left(\bar{q}+\bar{q}_{l}-1\right)} \\
& -\sum_{j=1}^{\bar{q}} \frac{4}{\left(\overline{r_{J}}+\overline{s_{J}}\right)\left(\overline{r_{j}}+\overline{s_{J}}-2\right)} \\
& \leq \frac{2}{n-2+\bar{q}}-\sum_{i=1}^{q-1} \frac{2}{\left(\bar{q}+\overline{q_{l}}\right)\left(\bar{q}+\overline{q_{l}}-1\right)} \\
& \leq \frac{2}{n-2+\bar{q}}-\sum_{i=1}^{q-1} \frac{2}{(\bar{q}+n-2)(\bar{q}+n-3)} \\
& =\frac{2}{n-2+\bar{q}}\left[1-\sum_{i=1}^{q-1} \frac{1}{(\bar{q}+n-3)}\right] \\
& =\frac{2}{n-2+\bar{q}}\left[1-\frac{q-1}{(\bar{q}+n-3)}\right] \\
& =\frac{2}{n-2+\bar{q}}\left[\frac{2 \bar{q}-1}{(n-3+\bar{q})}\right] \\
& <0 \quad[\text { Since } \quad \bar{q} \leq n-3]
\end{aligned}
$$

Therefore $\overline{H(G)}<\overline{H\left(G^{\prime}\right)}$. Hence the non-neighbor harmonic index of the graph $G$ strictly increases by the removal of pendant edge.

Corollary 1.1.1 Let $G$ be a graph with $n$ vertices and $m$ edges. Addition of a pendant edge to an arbitrary vertex of $G$, strictly decreases the non-neighbour harmonic index.

Definition 2.1. The graph $K_{n}+e$ is obtained by adding a pendant edge $e$ to any arbitrary vertex $K_{n}$.

Definition 2.2. For any edge $e=u v$ in the graph $G$, Weight of the edge $e$ is defined by the quantity $\frac{2}{\overline{d(u)}+\overline{d(v)}}[12]$.

Theorem 1.2. For the graph $K_{n}+e$, the non-neighbor harmonic index $\overline{H\left(K_{n}+e\right)}=\frac{2}{n-1}+\frac{(n-1)(n+2)}{2}$ and weight of the edge $e$ is $\frac{2}{n-1}$.

Proof. The number of non-neighbors of each vertex in $K_{n}$ is zero. Let $e=u v$ be the pendent edge attached to the arbitrary vertex $v$ of $K_{n}$. We have $\overline{d(u)}=n-1$ and $\overline{d(v)}=0$ so that the weight of the edge $e=u v$ 
is $\frac{2}{n-1}$. Remaining vertices $v_{1}, v_{2}, \ldots, v_{n-1}$ of $K_{n}$ has $\overline{d\left(v_{l}\right)}=1$. Therefore the non-neighbor harmonic index,

$$
\begin{gathered}
\overline{H\left(K_{n}+e\right)}=\frac{2}{n-1}+2(n-1)+\left(\begin{array}{l}
n \\
2
\end{array}\right)-(n-1) \\
=\frac{2}{n-1}+2(n-1)+\frac{n(n-1)}{2}-(n-1) \\
=\frac{2}{n-1}+\frac{(n-1)(n+2)}{2}
\end{gathered}
$$

Remark 1.2.1. The non-neighbor harmonic index of the complete graph $K_{n}$ is $\infty$. By adding an pendant edge to $K_{n}$, the non-neighbor harmonic index can be computed easily. The quantity $\frac{(n-1)(n+2)}{2}$ is the approximate value of the non-neighbor harmonic index of the complete graph $K_{n}$.

Theorem 1.3. Let $G$ be a weighted graph. Removal of an edge with maximal weight strictly decreases the nonneighbour harmonic index of the graph $G$.

Proof. Let $e=u v$ be an edge with maximal weight in $G$. Let $d(u)=p$ and $d(v)=q$ and hence $\overline{d(u)}=\bar{p}$ and $\overline{d(v)}=\bar{q}$ with $\bar{p}, \bar{q} \leq n-3$. Let $x_{1}, x_{2}, \ldots, x_{p-1}$ be the neighbors of $u$ other than $v$ and $y_{1}, y_{2}, \ldots, y_{q-1}$ be the neighbours of $v$ other than $u$. Let $G^{\prime}=G-e$ be a connected graph then we have $\bar{p}+\bar{q} \leq \bar{p}+\overline{d\left(x_{l}\right)}$ and $\bar{p}+\bar{q} \leq \bar{q}+\overline{d\left(y_{l}\right)}$. Hence

$$
\begin{aligned}
\overline{H(G)}-\overline{H\left(G^{\prime}\right)}= & \frac{2}{\bar{p}+\bar{q}}+\sum_{i=1}^{p-1} \frac{2}{\bar{p}+\overline{d\left(x_{l}\right)}} \\
& -\sum_{i=1}^{p-1} \frac{2}{\bar{p}+\overline{d\left(x_{l}\right)}+1}+\sum_{j=1}^{q-1} \frac{2}{\bar{q}+\overline{d\left(y_{J}\right)}} \\
=\frac{2-1}{\bar{p}+\bar{q}}-\sum_{i=1}^{p-1} \frac{2}{\left(\bar{p}+\overline{d\left(x_{l}\right)}\right)\left(\bar{p}+\overline{d\left(x_{l}\right)}+1\right)} & 2 \\
& +\sum_{j=1}^{q-1} \frac{2}{\left(\bar{q}+\overline{d\left(y_{J}\right)}\right)\left(\bar{q}+\overline{d\left(y_{J}\right)}+1\right)}
\end{aligned}
$$

$$
\begin{aligned}
& \geq \frac{2}{\bar{p}+\bar{q}}+\sum_{i=1}^{p-1} \frac{2}{(\bar{p}+\bar{q})(\bar{p}+\bar{q}+1)} \\
& \quad+\sum_{j=1}^{q-1} \frac{2}{(\bar{p}+\bar{q})(\bar{p}+\bar{q}+1)} \\
& =\frac{2}{\bar{p}+\bar{q}}+\frac{2(p-1)}{(\bar{p}+\bar{q})(\bar{p}+\bar{q}+1)}+\frac{2(q-1)}{(\bar{p}+\bar{q})(\bar{p}+\bar{q}+1)} \\
& =\frac{2}{\bar{p}+\bar{q}}+\frac{2 p+2 q-4}{(\bar{p}+\bar{q})(\bar{p}+\bar{q}+1)} \\
& >0
\end{aligned}
$$

Therefore $\overline{H(G)}>\overline{H\left(G^{\prime}\right)}$. Hence the non-neighbor harmonic index of the graph $G$ strictly decreases by the removal of an edge with maximal weight.

Corollary 1.3.1. Let $G$ be a weighted graph. Removal of an edge with minimal weight which is not a pendant edge, strictly decreases the non-neighbour harmonic index.

\section{(B) The non-neighbor harmonic index for the subdivision of graphs}

In this section the non-neighbor harmonic index for the subdivision of some standard graphs are computed and the relation between non-neighbor harmonic index of the graph $G$ and its subdivision graph $S(G)$ is discussed.

Theorem 2.1. For the $k$-regular graph $G$ where $k \geq 2$, $\overline{H(S(G))}=\frac{2 n k}{n k+2 n-k-4}$

Proof. A $k$-regular graph has $n$ vertices and $\frac{n k}{2}$ edges. The non-neighbors of each vertex is $(n-k-1)$. Hence for a $k$-regular graph $G, \overline{H(G)}=\frac{n k}{2(n-k-1)}$. The subdivision graph $S(G)$ of a $k$-regular graph $G$ has $n+\frac{n k}{2} \quad$ vertices and $n k$ edges. Each vertex in the $k$-regular graph has $\left(\frac{n k}{2}+n-k-\right.$ 1) non-neighbors in $S(G)$ and each vertex obtained by subdividing the edges of $G$ has $\left(\frac{n k}{2}+n-3\right)$ non neighbors. Hence

$$
\begin{gathered}
\overline{H(S(G))}=n k\left[\frac{2}{\frac{n k}{2}+n-k-1+\frac{n k}{2}+n-3}\right] \\
=n k\left[\frac{2}{n k+2 n-k-4}\right] \\
=\frac{2 n k}{n k+2 n-k-4}
\end{gathered}
$$

Corollary 2.1.1. For the complete graph $K_{n}(n \geq 2)$, $\overline{H\left(S\left(K_{n}\right)\right)}=\frac{2 n(n-1)}{n^{2}-3}$. 
Corollary 2.1.2. For the cycle $C_{n}(n \geq 2), \overline{H\left(S\left(C_{n}\right)\right)}=$ $\overline{H\left(C_{2 n}\right)}=\frac{2 n}{2 n-3}$.

Theorem 2.2. For the path $P_{n}(n \geq 3), \overline{H\left(S\left(P_{n}\right)\right)}=$ $\overline{H\left(P_{2 n-1}\right)}=\frac{4}{4 n-7}+1$.

Proof: The non-neighbor harmonic index of the path $P_{n}(n \geq 4)$ is $\overline{H\left(P_{n}\right)}=\frac{4}{2 n-5}+1$ [13]. The subdivision of path $P_{n}$ is the path with $(2 n-1)$ vertices. Hence

$$
\begin{aligned}
\overline{H\left(S\left(P_{n}\right)\right)} & =\overline{H\left(P_{2 n-1}\right)} \\
& =\frac{4}{2(2 n-1)-5}+1 . \\
& =\frac{4}{4 n-7}+1 .
\end{aligned}
$$

Note: $\overline{H\left(S\left(P_{2}\right)\right)}=\overline{H\left(P_{3}\right)}=4$

Theorem 2.3. For the complete bipartite graph $K_{m, n}$, $\overline{H\left(S\left(K_{m, n}\right)\right)}=m n\left[\frac{2}{2 m(n+1)+n-4}+\frac{2}{2 n(m+1)+m-4}\right]=$ $\overline{H\left(K_{m, n}\right)}\left[\frac{m+n-2}{2 m(n+1)+n-4}+\frac{m+n-2}{2 n(m+1)+m-4}\right]$.

Proof: The non-neighbor harmonic index of the complete bipartite graph $K_{m, n}$ is $\overline{H\left(K_{m, n}\right)}=\frac{2 m n}{m+n-2} \quad$ [13]. The subdivision of a complete bipartite graph has $(m+n+m n)$ vertices and $2 m n$ edges. The non-neighbors of the vertices in the vertex set $V_{1}$ is $(m-1+m n)$ and the non-neighbors of the vertices in the vertex set $V_{2}$ is $(n-1+m n)$. The vertices which subdivide the edges are of degree 2 and their number of non-neighbors is $(m+n+m n-3)$. Hence

$$
\begin{aligned}
& \overline{H\left(S\left(K_{m, n}\right)\right)}=m n\left[\frac{2}{m-1+m n+m+n+m n-3}\right. \\
& \left.+\frac{2}{m+n+m n-3+n-1+m n}\right] \\
& =m n\left[\frac{2}{2 m(n+1)+n-4}+\frac{2}{2 n(m+1)+m-4}\right] \\
& =\frac{2 m n}{m+n-2}\left[\frac{m+n-2}{2 m(n+1)+n-4}+\frac{m+n-2}{2 n(m+1)+m-4}\right] \\
& =\overline{H\left(K_{m, n}\right)}\left[\frac{m+n-2}{2 m(n+1)+n-4}+\frac{m+n-2}{2 n(m+1)+m-4}\right] .
\end{aligned}
$$

Corollary 2.3.1. For the $k$-regular bipartite graph, $\overline{H\left(S\left(K_{k, k}\right)\right)}=\frac{4 k^{2}}{2 k^{2}+3 k-4}=\overline{H\left(K_{k, k}\right)}\left[\frac{4(k-1)}{2 k^{2}+3 k-4}\right]$
Corollary 2.3.2. For the star $S_{k}$,

$\overline{H\left(S\left(S_{k}\right)\right)}=k\left[\frac{2}{3 k-2}+\frac{2}{4 k-3}\right]=\overline{H\left(S_{k}\right)}\left[\frac{k-1}{3 k-2}+\frac{k-1}{4 k-3}\right]$

Theorem 2.4. For the wheel $W_{n}(n \geq 4), \overline{H\left(S\left(W_{n}\right)\right)}=$ $\left[\frac{6 n}{6 n-5}+\frac{2 n}{5 n-2}\right]=\overline{H\left(W_{n}\right)}+\left[\frac{36 n-37 n^{2}-48 n^{3}}{(6 n-5)(5 n-2) 9 n-3)}\right]$.

Proof: The non-neighbor harmonic index of the Wheel $W_{n}$, is $\overline{H\left(W_{n}\right)}=\frac{3 n}{n-3}$ [13]. The subdivision of wheel graph has $(3 n+1)$ vertices and $4 n$ edges. The vertices which lie on the cycle $C_{n}$ has $(3 n-3)$ non-neighbors and the vertices which subdivide the $2 n$ edges has $(3 n-2)$ non-neighbors. The vertex at the center has $2 n$ non-neighbors. Hence

$$
\begin{gathered}
\overline{H\left(S\left(W_{n}\right)\right)}=3 n\left[\frac{2}{3 n-3+3 n-2}\right]+n\left[\frac{2}{2 n+3 n-2}\right] \\
=\left[\frac{6 n}{6 n-5}+\frac{2 n}{5 n-2}\right] \\
=\frac{3 n}{n-3}+\left[\frac{6 n}{6 n-5}+\frac{2 n}{5 n-2}-\frac{3 n}{n-3}\right] \\
=\overline{H\left(W_{n}\right)}+\left[\frac{36 n-37 n^{2}-48 n^{3}}{(6 n-5)(5 n-2) 9 n-3)}\right] .
\end{gathered}
$$

Note: $\overline{H\left(S\left(W_{3}\right)\right)}=\frac{24}{13}$

(C) The Non-Neighbor Harmonic Index for the Tadpole graph, Ladder graph and their subdivision graphs.

A tadpole graph $T_{n, k}[14,15,16]$ is a graph obtained by joining a cycle $C_{n}(n \geq 3)$ to a path of length $k$ and a ladder graph $L_{n}=K_{2} \times P_{n}$ where $P_{n}$ is a path with $n$ vertices, $K_{2}$ is a complete graph with two vertices and $\times$ denotes the cartesian product. When $n=1, L_{n}$ is a path of length 1 . When $n=2, L_{n}$ is a cycle $C_{4}$. The non-neighbor harmonic index for the tadpole graph $T_{n, k}$ and the ladder graph $L_{n}$ together with their subdivision graphs are provided in this section.

Theorem 3.1. For the tadpole graph $T_{n, k}$,

$$
\begin{aligned}
& \overline{\mathrm{H}\left(T_{n, k}\right)}= \\
& \left\{\begin{array}{lr}
\frac{1}{n-2}+\overline{H\left(P_{n}\right)} & \text { if } k=1 \text { and } n \geq 4 \\
\frac{2}{2 n+2 k-5}+\frac{2 n+2 k-8}{2 n+2 k-6}+\frac{6}{2 n+2 k-7} & \text { if } k \geq 2
\end{array}\right.
\end{aligned}
$$

Proof: A tadpole graph $T_{n, k}$ has $n+k$ vertices and $n+k$ edges.

Case(i) If $k=1$, Then the tadpole graph $T_{n, 1}$ has $(n+1)$ vertices and $(n+1)$ edges. The pendant vertex in $T_{n, 1}$ has $(n-1)$ non-neighbors and the vertex attachd to the cycle $C_{n}$ has $(n-3)$ non-neighbors. The remaining vertices in the cycle $C_{n}$ has $(n-2)$ non-neighbors. Hence 


$$
\begin{aligned}
\overline{H\left(T_{n, 1}\right)} & =\left[\frac{2}{n-1+n-3}\right]+\left[\frac{4}{n-3+n-2}\right] \\
& \quad+(n-2)\left[\frac{2}{n-2+n-2}\right] \\
& =\frac{2}{2 n-4}+\frac{4}{2 n-5}+1 \\
& =\frac{1}{n-2}+\overline{H\left(P_{n}\right)}
\end{aligned}
$$

Case(ii) Suppose $k \geq 2$, then $T_{n, k}$ has $(n+k-2)$ vertices of degree 2 , one vertex of degree 3 and a pendant vertex. The pendant vertex in the path $P_{n}$ has $(n+k-2)$ non-neighbors and the vertex of $P_{n}$ attached to the cycle $C_{n}$ has $(n+k-4)$ non-neighbors. The remaining vertices each have $(n+k-$ 3) non-neighbors. Hence

$$
\begin{gathered}
\overline{H\left(T_{n, k}\right)}=\left[\frac{2}{n+k-2+n+k-3}\right]+(k-2)\left[\frac{2}{n+k-3+n+k-3}\right]+ \\
{\left[\frac{6}{n+k-4+n+k-3}\right]+(n-2)\left[\frac{2}{n+k-3+n+k-3}\right]} \\
=\frac{2}{2 n+2 k-5}+\frac{2 n+2 k-8}{2 n+2 k-6}+\frac{6}{2 n+2 k-7}
\end{gathered}
$$

Note: $\overline{H\left(T_{3,1}\right)}=6$

Theorem 3.2. For the tadpole graph $T_{n, k}$,

$\overline{H\left(S\left(T_{n, k}\right)\right)}=\overline{H\left(S\left(T_{2 n, 2 k}\right)\right)}=\frac{2}{4 n+4 k-5}+\frac{4 n+4 k-8}{4 n+4 k-8}+\frac{6}{4 n+4 k-7}$

\section{Proof:}

The subdivision of the tadpole graph $S\left(T_{n, k}\right)$ is the tadpole graph with a cycle of $2 n$ vertices attached to the path of length $2 k$. Hence

$$
\begin{gathered}
\overline{H\left(S\left(T_{n, k}\right)\right)}=\overline{H\left(T_{2 n, 2 k}\right)} \\
=\frac{2}{4 n+4 k-5}+\frac{6 n+4 k-8}{4 n+4 k-8}+\frac{6}{4 n+4 k-7}
\end{gathered}
$$

Theorem 3.3. For the ladder graph $L_{n}$ where $n \geq 3$,

$$
\overline{H\left(L_{n}\right)}=\frac{8}{4 n-7}+\frac{3 n-8}{2(n-2)}+\frac{2}{2 n-3}
$$

Proof. The ladder graph has $2 n$ vertices and $(3 n-2)$ edges among which $(2 n-4)$ vertices are of degree 3 and four vertices are of degree 2 . The number of non-neighbors of the 4 corner vertices is $(2 n-3)$ and the remaining $(2 n-4)$ vertices has $(2 n-4)$ non neighbors. Hence

$$
\begin{aligned}
& \overline{H\left(L_{n}\right)}=4\left[\frac{2}{2 n-3+2 n-4}\right] \\
& +2(n-3)\left[\frac{2}{2 n-4+2 n-4}\right] \\
& +2\left[\frac{2}{2 n-3+2 n-3}\right] \\
& +(n-2)\left[\frac{2}{2 n-4+2 n-4}\right] \\
& =\frac{8}{4 n-7}+\frac{4 n-12+2 n-4}{4 n-8}+\frac{4}{4 n-6} \\
& =\frac{8}{4 n-7}+\frac{6 n-16}{4(n-2)}+\frac{4}{4 n-6} \\
& =\frac{8}{4 n-7}+\frac{3 n-8}{2(n-2)}+\frac{2}{2 n-3}
\end{aligned}
$$

Theorem 3.4. For the ladder graph $L_{n}$ where $n \geq 3$, $\overline{H\left(S\left(L_{n}\right)\right)}=\frac{16}{10(n-1)}+\frac{12(n-2)}{10 n-11}$

Proof. The subdivision of a ladder graph $S\left(L_{n}\right)$ has $(5 n-$ $2)$ vertices and $(6 n-4)$ edges. Among the $(5 n-2)$ vertices $2(n-2)$ vertices has degree 3 and the remaining vertices have degree 2 . Hence the number of non-neighbores of these vertices $2(n-2)$ vertices is $(5 n-6)$ and that of remaining vertices is $(5 n-5)$. Hence

$$
\begin{aligned}
\overline{H\left(S\left(L_{n}\right)\right)}= & 8\left[\frac{2}{5 n-5+5 n-5}\right] \\
& \quad+(6 n-12)\left[\frac{2}{5 n-5+5 n-6}\right] \\
= & \frac{16}{10(n-1)}+\frac{12(n-2)}{10 n-11}
\end{aligned}
$$

\section{CONCLUSION AND FUTURE SCOPE}

In this paper we have presented in detail the behaviour of non-neighbor harmonic index on elementary graph operations. As future work study on the behaviour of nonneighbor harmonic index on advanced graph operations could be carried out.

\section{REFERENCES}

[1] James Devillers and Alexandru T.Balaban, "Topological Indices and Related Descriptors in QSAR and QSPAR", Gordon and Breach Science Publishers.

[2] Ivan Gutman, "Degree-based topological indices", Croat. Chem. Acta, 86(4)(2013), 351-361.

[3] Kexiang $\mathrm{Xu}$, Muhuo Liu, Kinkar Ch.Das, Ivan Gutman, Boris Furtula, "A Survey on Graphs Extremal with Respect to DistanceBased Topological Indices", MATCH Commun. Math. Comput. Chem. 71(2014), 461-508.

[4] Huiqing Liu, Mei Lu, Feng Tian, "On the Randic index", Journal of Mathematical Chemistry Vol. 38, No. 3, October (2005).

[5] Martin Knor, Riste Skrekovski, Aleksandra Tepeh, "Mathematical aspects of Wiener index", arXiv:1510.00800v1 [math.CO] 3 Oct (2015). 
[6] Ivan Gutman, Boris Furtula, Zana Kovijanic Vukicevic and Goran Popivoda, "On Zagreb Indices and Coindices", MATCH Commun. Math. Comput. Chem., 74(2015), 5-16.

[7] Douhlas B.West, "Introduction to Graph Theory", Second Edition, PHI Learning Private Limited, New Delhi.

[8] Shaoqiang Liu, Jianxi Li, "Some Properties on the Harmonic Index of Molecular Trees", Hindawi Publishing Corporation, ISRN Applied Mathematics, Volume 2014, Article ID 781648.

[9] Lingping Zhong, "The harmonic index for Graphs", Applied Mathematics Letters, 25(2012)561-566.

[10] Juan Carlos Hernandez-Gomez, Jesus Romero-Valencia, R. Reyes Carreto, "Mathematical Aspects on the Harmonic Index", International Journal of Mathematical Analysis, Vol. 11, (2017), no. 2, 85 - 95 .

[11] A.Rizwana, G.Jeyakumar, S.Somasundaram, "Non-Neighbor Topological Indices for Hydrocarbons", International Journal of Scientific Engineering and Science, Volume 1, Issue 7, pp. 16-19, 2017.

[12] Aleksandar Ilic, "Note on the harmonic index of a Graph", arXiv:1204.3313v1 [math.CO] 15 Apr (2012).

[13] A.Rizwana, G.Jeyakumar, S.Somasundaram, "On the Non-Neighbor Zagreb Indices and Non-Neighbor Harmonic Index", International Journal of Mathematics and its Applications, Volume 4, Issue 2-D (2016), 89-101.

[14] Bindusree, V.Lokesha, P.S.Ranjini, "Relation connecting Zagreb co-indices on Three graph Operators", Mathematica Aeterna, Vol.3, 2013, no. 6, 433-448.

[15] K.Dhanalakshmi, J.Amalorpava Jerline, L.Benedict Michaelraj, "Modified and Multiplicative Zagreb Indices on Graph Operators", Journal of Computer and Mathematical Sciences, Vol.7(4), 225-232, April 2016.

[16] P.S.Ranjini, V.Lokesha, "Smarandache-Zagreb Index on Three Graph Operators", International J.Math. Combin. Vol.3(2010), 01-10.

\section{AUTHORS PROFILE}

Ms.A.Rizwana completed Bachelor of Science from Sadakathullah Appa College, affiliated to Manonmaniam Sundaranar University, Tirunelveli-12, Tamilnadu in 2006 and Master of Science from Sarah Tucker College, affiliated to Manonmaniam Sundaranar University in 2008. She completed her Master of Philosophy from St.Xaviers College (Autonomous) in 2009. She is currently pursuing Ph.D and working as Head \& Assistant Professor in Department of Mathematics (Unaided), Sadakathullah Appa College (Autonomous) since 2014. She has cleared her State Eligibility Test (SET)-Tamilnadu in 2016. She has published more than 5 research papers in reputed international journals and presented several papers in national and international conferences. Her main research work focuses on Topological Indices in Chemical Graph Theory. She has 8 years of teaching experience and 4 years of Research Experience.

Dr.G.Jeyakumar is working as Associate Professor and Head, Department of Mathematics, St.John's College, Affiliated to Manonmaniam Sundaranar University, Tirunelveli. He completed his Undergraduate, Postgraduate and M.Phil in Mathematics from Manonmaniam Sundaranar University, Tirunelveli, Tamilnadu. $\mathrm{He}$ has completed his $\mathrm{PhD}$ from Alagappa University, Karaikudi. He has involved in various academic activities. He has more than 10 research publications in reputed journals. He has presented several research papers in national and international conferences. He has guided more than 5 research scholars in which two of them have completed their Doctoral degree. He has got 34 years of teaching experience. His area of specialization is Graph Labeling in Graph Theory. 